\title{
construckián de un anfiteatro subterráneo de mil plazas
}

J. M. HERENG, ingeniero

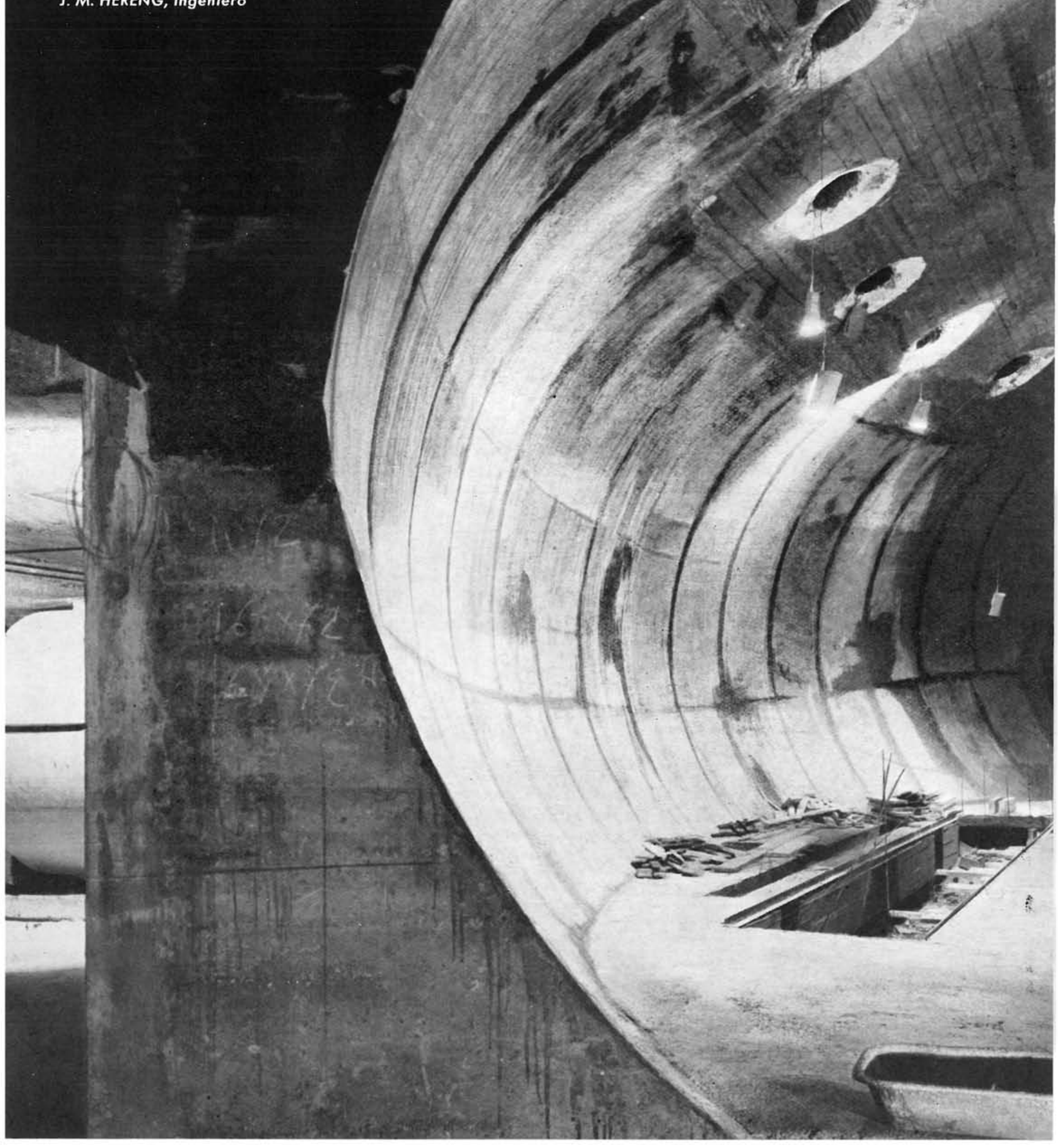


Colocación de armaduras en la parte inferior.

Hormigonando la parte inferior.

$146-64$

\section{simopsis}

El Conservatorio de Artes y Oficios de Paris necesitaba un anfiteatro capaz para mil plazas, utilizable para clases y conferencias. Por falta de espacio se deciaío construirlo subterraneo en uno de os patios.

La solución adoptada fue la de un elipsoide de revolución de eje mayor inclinado respecto de la horizontal con $32 \mathrm{~m}$ de diámetro

Esta solución es de particular interés, ya que solventaba de una vez dos problemas: el de cimentación, que, en general, plantean todos los terrenos de relleno, y el de no restar iluminación a los cuerpos y edificios circundantes. La carga sobre el suelo es siempre inferior a 380 gramos por centímetro cuadrado, aun en el supuesto accidental de un asiento local en cualquier zona de apoyo de $20 \mathrm{~m}$ de diámetro. El transporte del hormigón se ha realizado por canalizaciones de aire comprimido a fin de no entorpecer el ritmo de hormigonar. Los encofrados han sido detenidamente estudiados, en particular los de la zona
bóveda que cubre el auditorio.

Los entramados tubulares que sostienen los encofrados de dicha bóveda se han montado a base de montantes normales al plano diametral inclinado. Con esta disposicion ouriosa, el personal que calidad $y$, con ello, el del equilibrio de su propia persona.

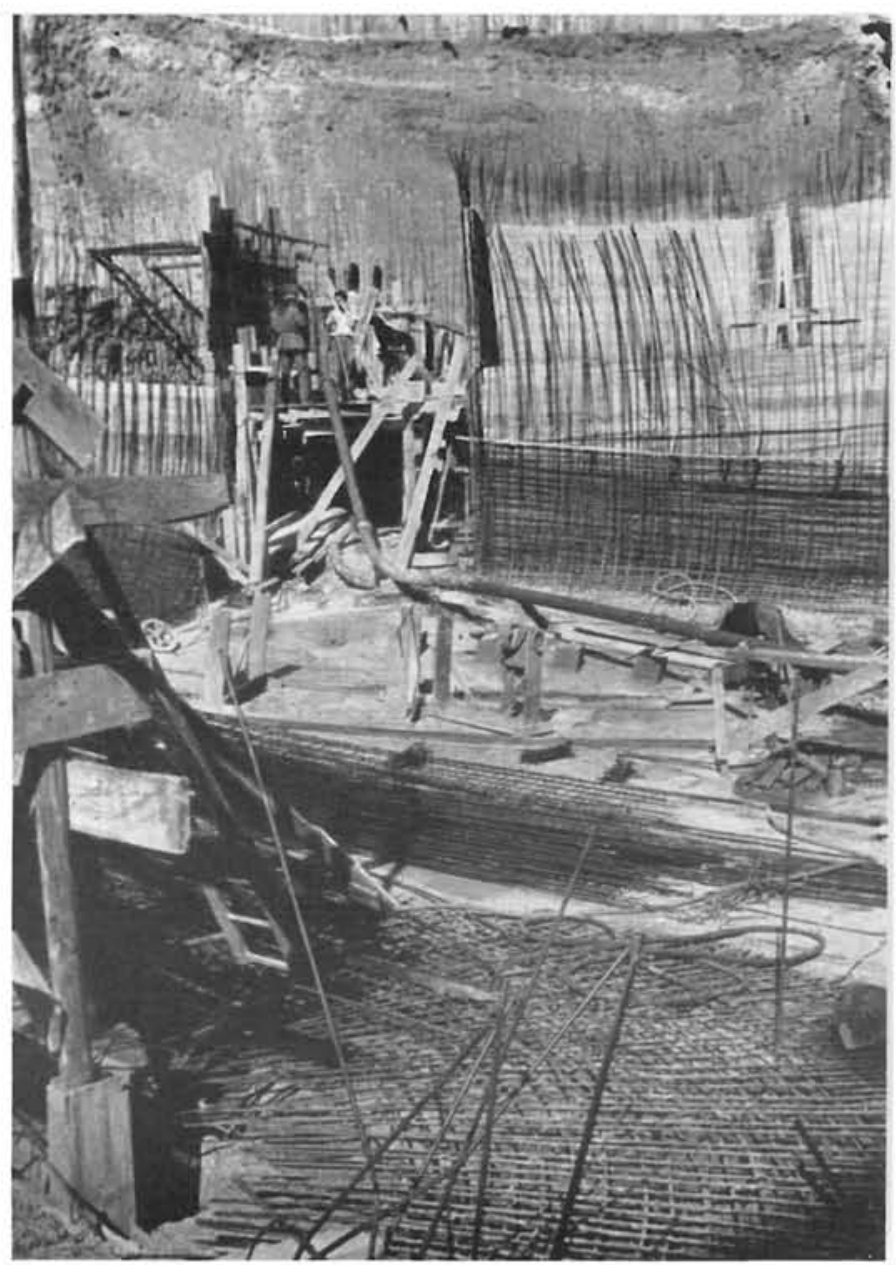

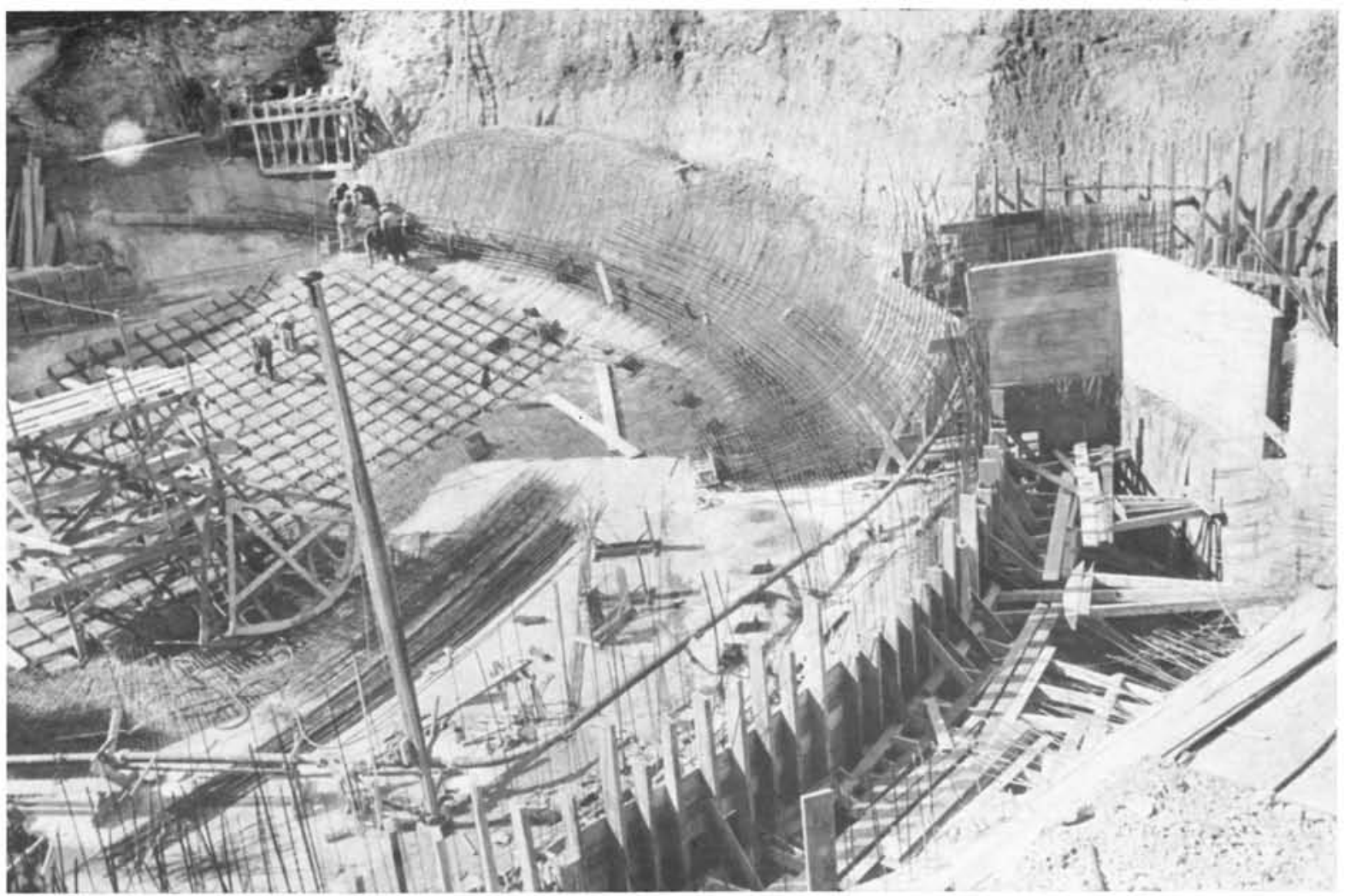


p a
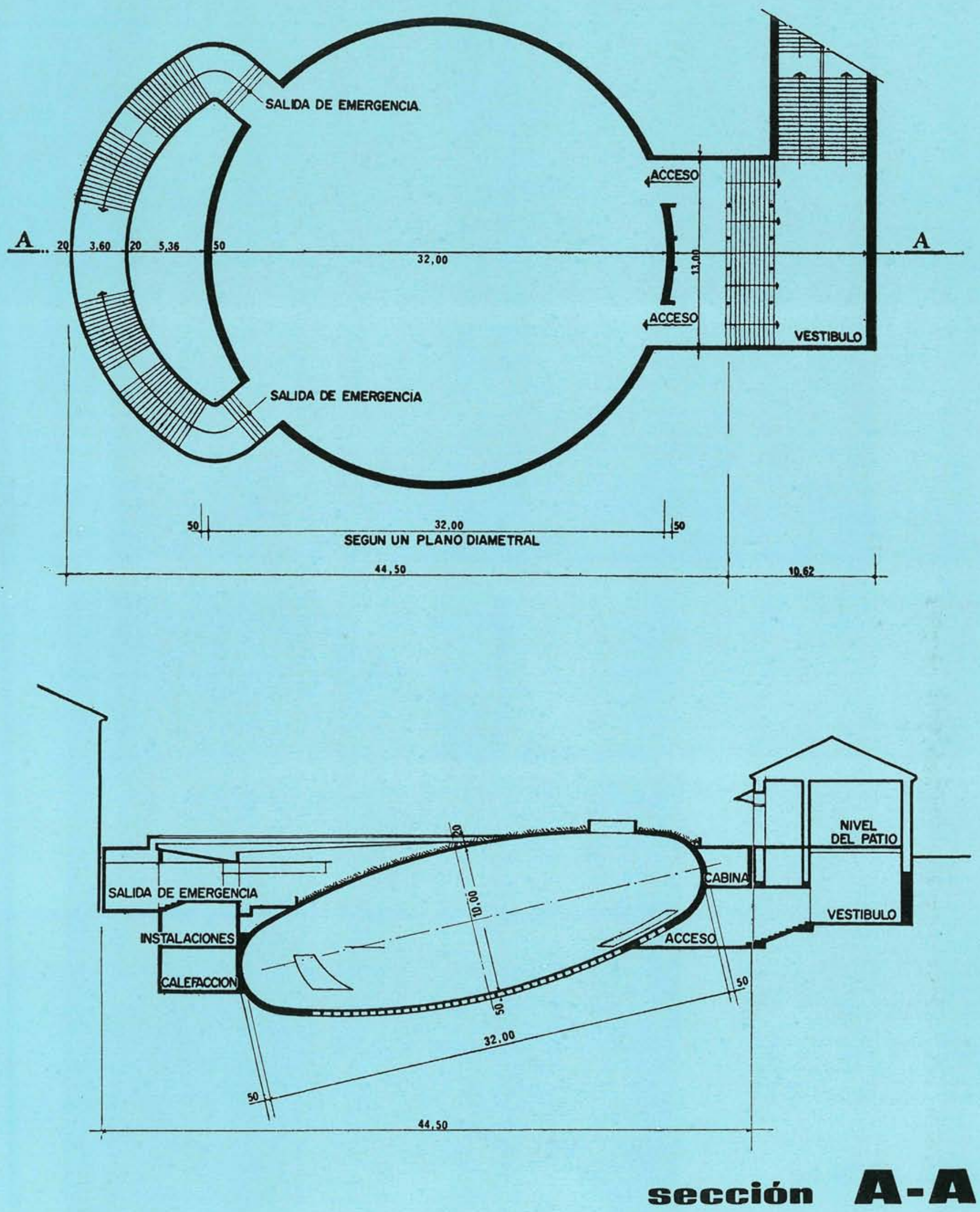


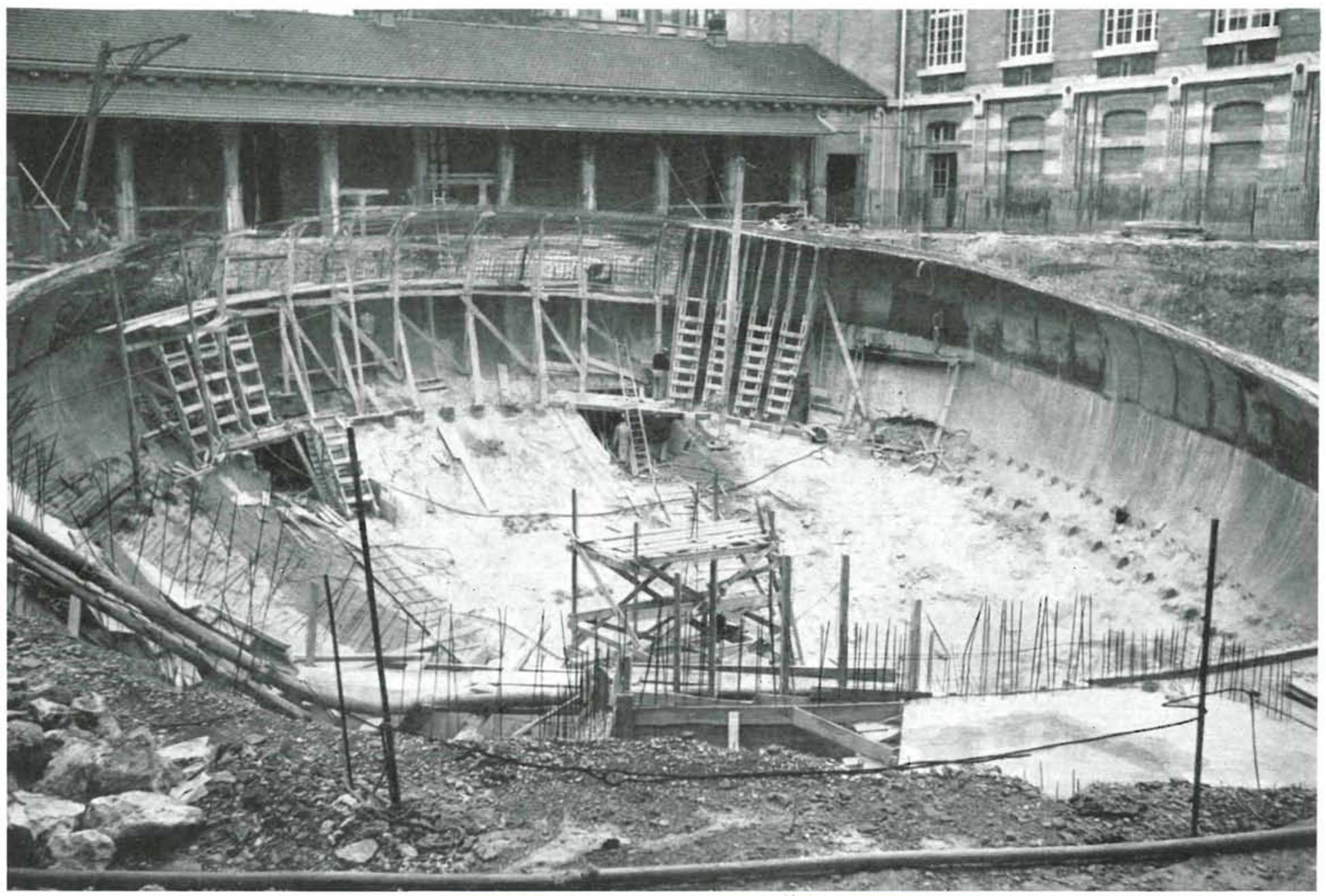

Hormigonado de la zona tórica.

La Escuela Nacional de Artes y Oficios de París, conocida con el nombre de Conservatorio de Artes y Oficios, es un Centro dedicado a la enseñanza técnica de tipo medio, que necesitaba un anfiteatro con capacidad de mil plazas, ya que el número de alumnos es numeroso.

Con objeto de no disminuir la iluminación natural del resto del edificio se decidió construir un anfiteatro subterráneo bajo la superficie de uno de los patios del actual edificio. Como el terreno se compone de un relleno de canteras explotadas anteriormente, el problema de cimientos creaba una serie de inconvenien. tes que debían estudiarse antes de tomar una decisión.

Llegar hasta el firme suponía descender bastante $y$ apoyarse en pilares que se construirían abriendo pocillos; el vaciado total resultaba excesivo y la cons.

Encofrado de la parte tórica.

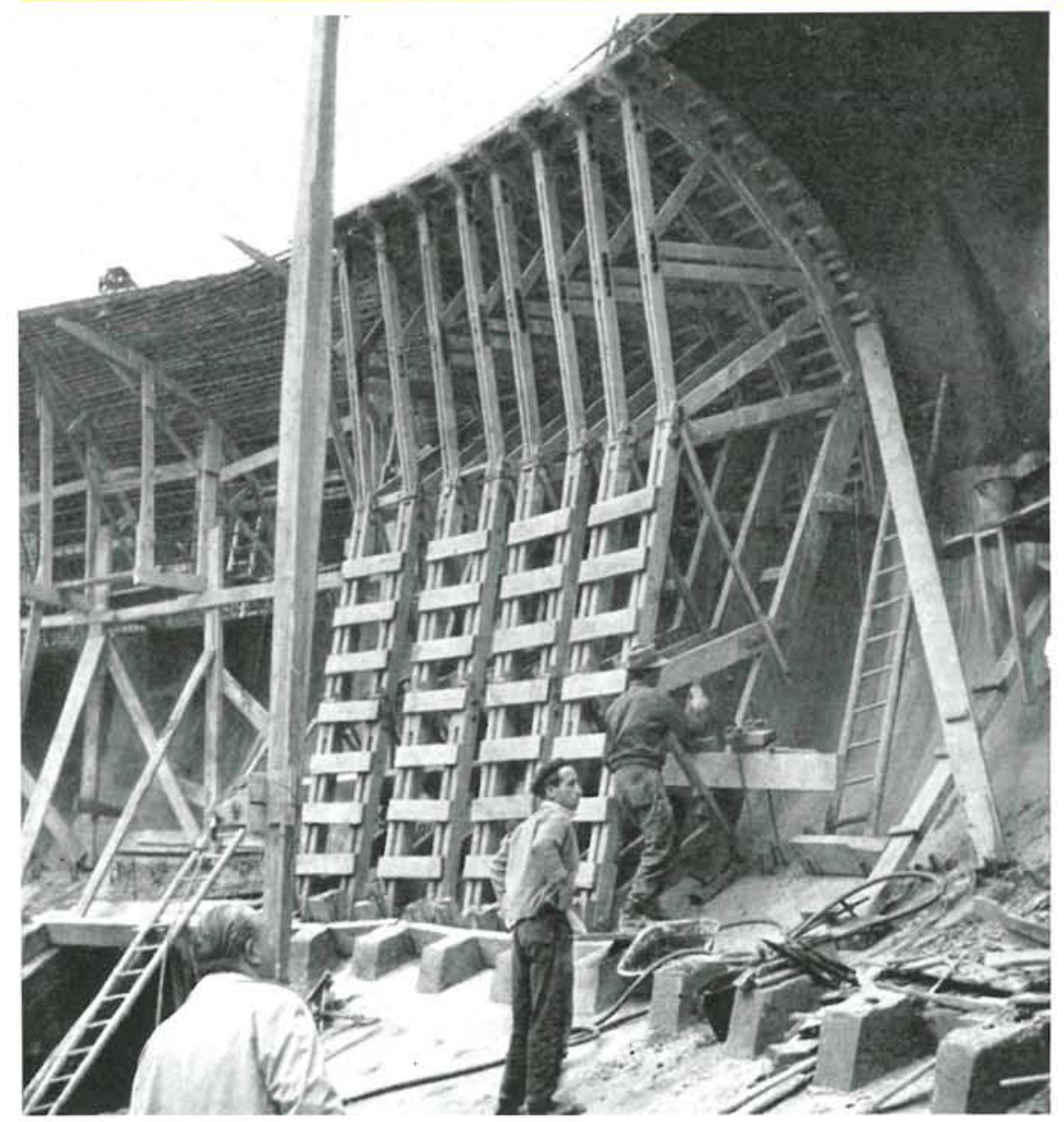


Superficie desencofrada.

Entramado tubular inclinado.

Preparación del encofrado superior.

Formación de escaños y plataformas.

trucción de una gran losa armada de apoyo especie de solera, encarecía considerablemen te el proyecto. Todo ello hizo pensar en una solución subterránea, exigiendo condiciones capaces de prever asientos diferenciales de gran amplitud. La solución final recayó en una superficie ovoide cuya parte inferior se adaptase a la rampa de una platea, y la superior, desprovista de soportes, en forma de perver, dos espesores de este, en forma de provocado por las tierras que rodean al óvulo.

Esta solución sencilla presenta no pocas dificultades en su ejecución $\mathrm{y}$ disposición general, ya que la iluminación será forzosamente artificial, se ha de ventilar, proporcio nar acceso amplio y contar con vestíbulo y escaleras de emergencia de capacidad sufi. ciente para prevenir a las exigencias de la seguridad.

La estructura enterrada es un elipsoide de revolucion, con eje mayor inclinado respecto de la horizontal, y el normal a este es un eje de revolución inclinado respecto de la vertical. En el plano inclinado que contiene al eje mayor, es decir en la sección del elipsoide, la forma una circunferencia de $32 \mathrm{~m}$ tórica rametron yaltura tota refor eje longitudinal segun una sección producida por un nlano vertical, es de $10 \mathrm{~m}$. El espesor de la parte inferior de apoyo sobre el terreno es de $0,50 \mathrm{~m}$, uniforme hasta llegar a la zona

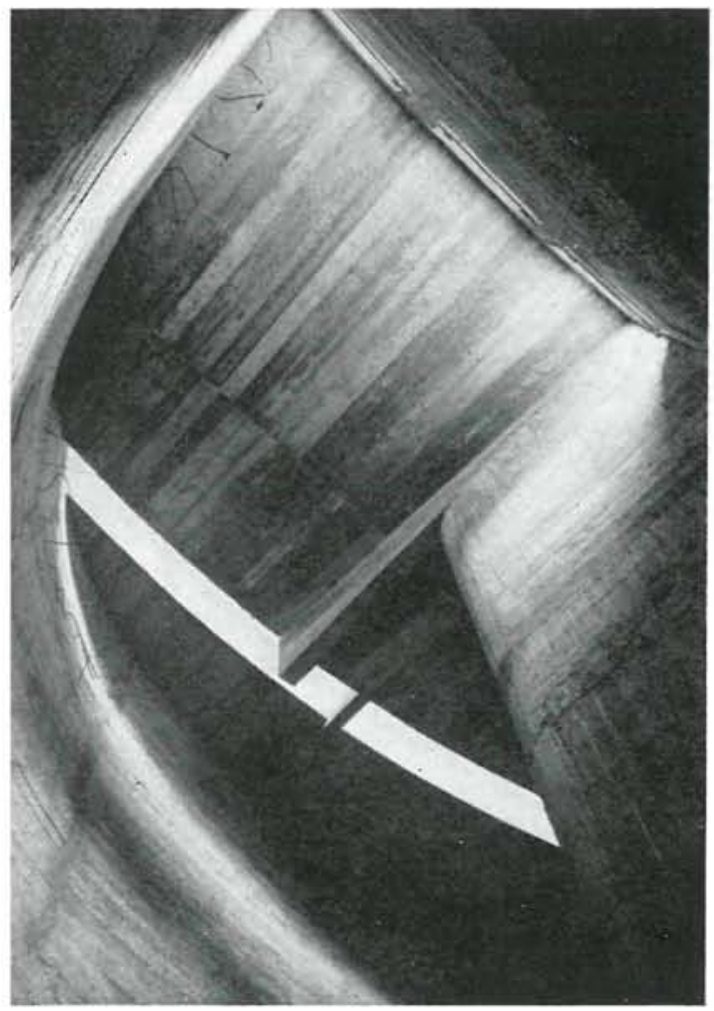

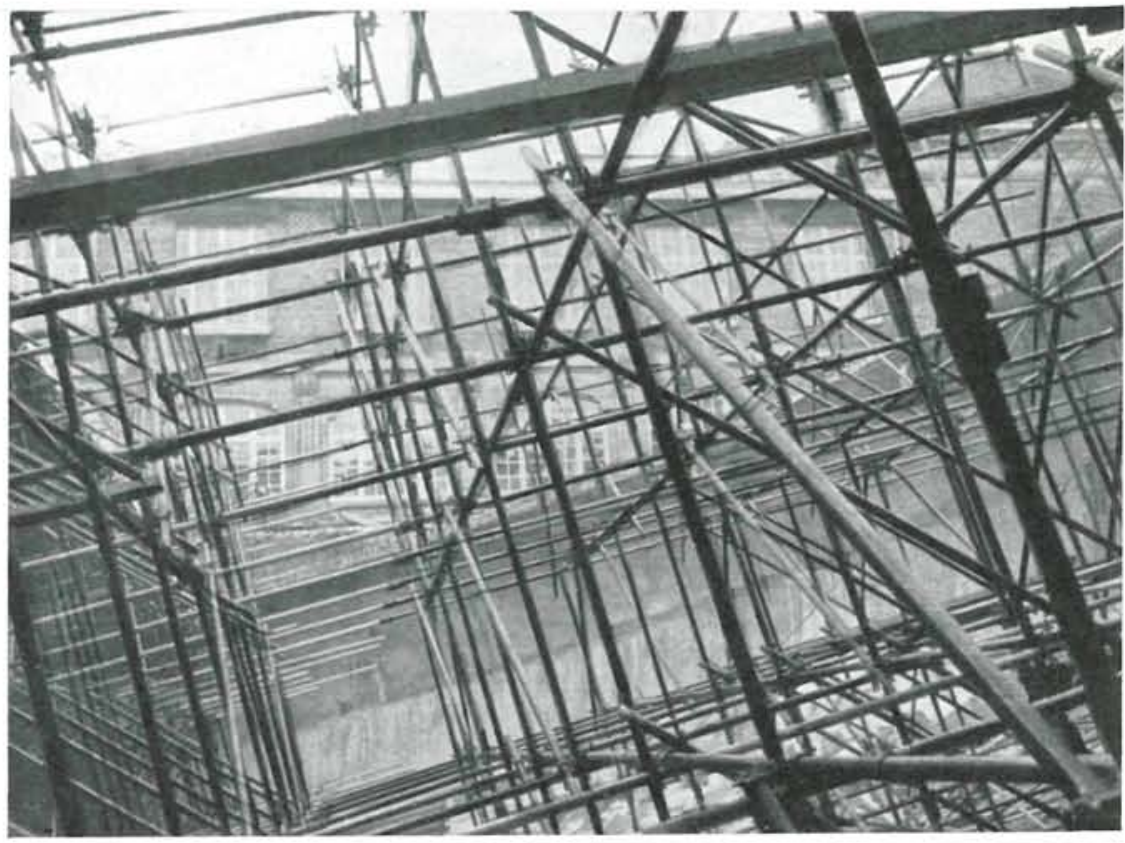
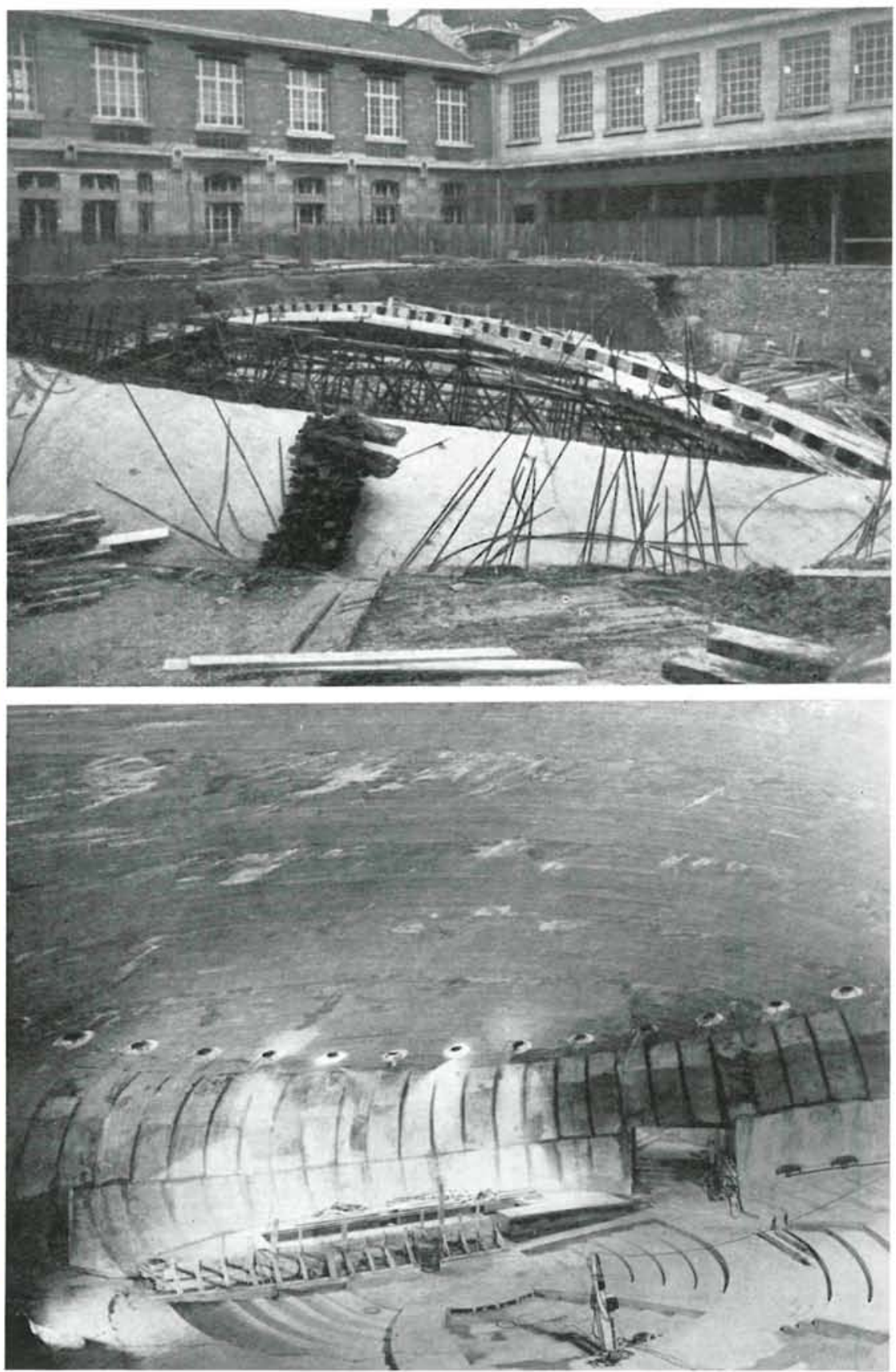

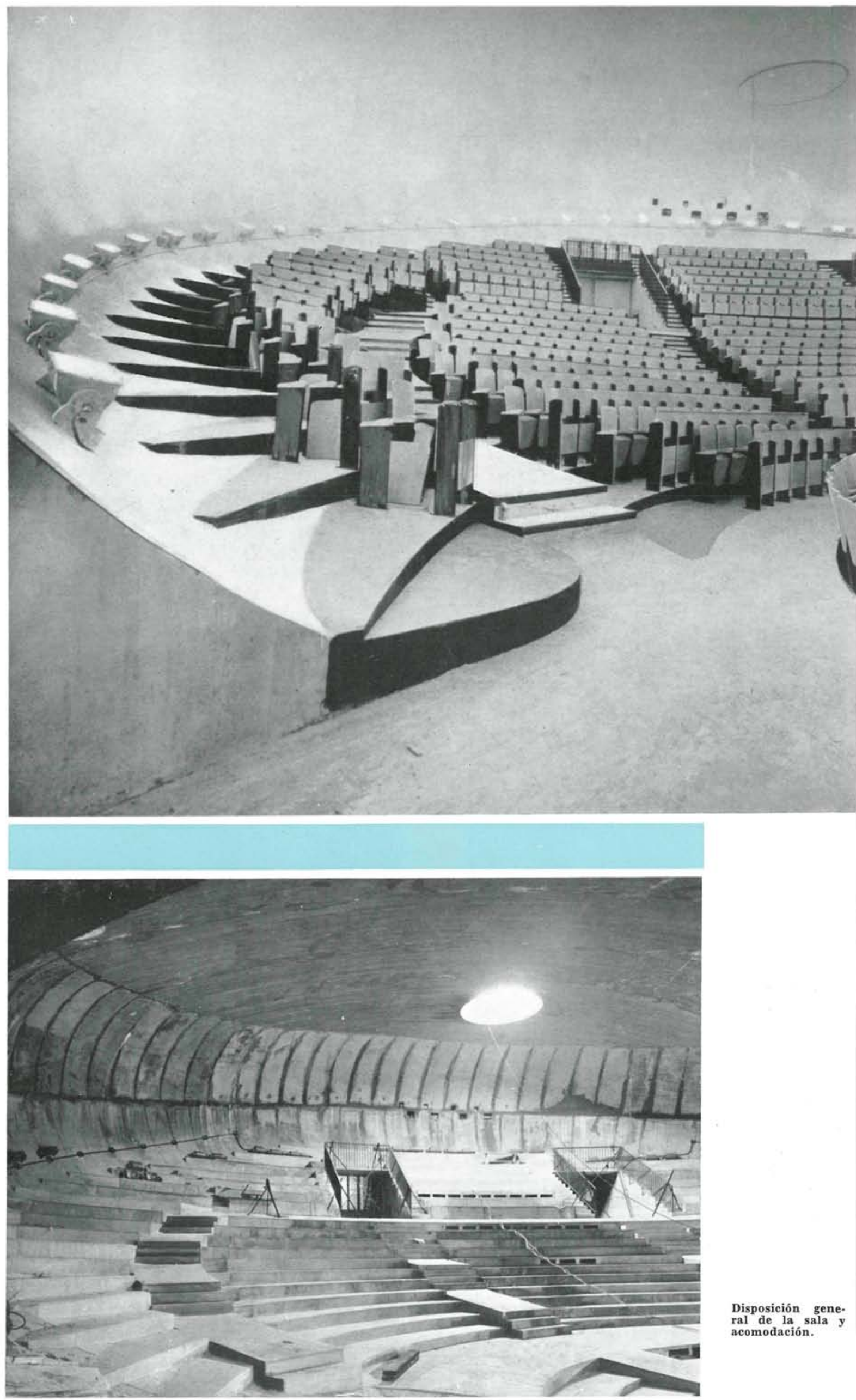

Disposición gene-
ral de la sala y ral de la sala 

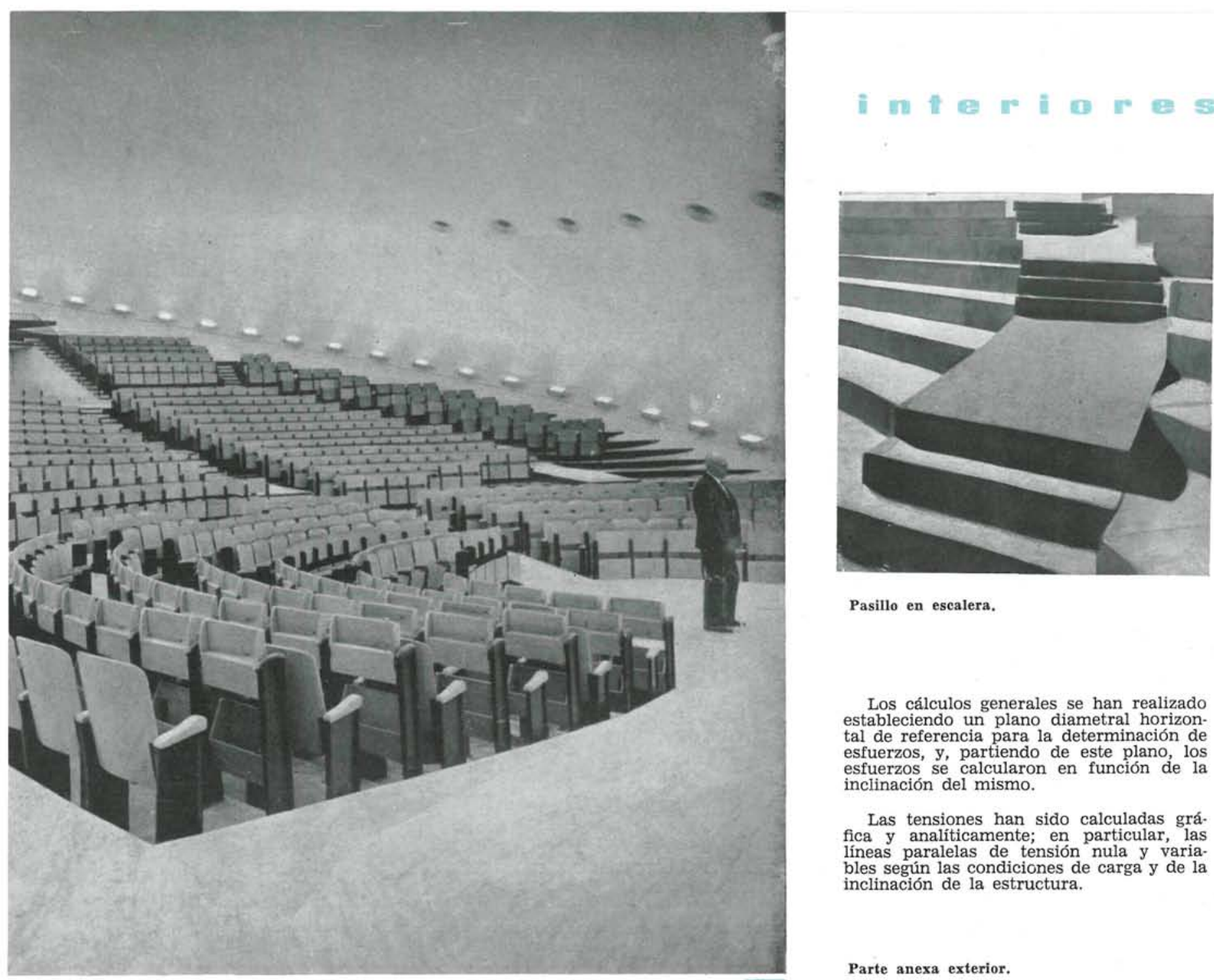

Pasillo en escalera.

El anfiteatro terminado. estableciendo un plano diametral horizontal de referencia para la determinación de esfuerzos, y, partiendo de este plano, los esfuerzos se calcularon en función de la inclinación del mismo.

Las tensiones han sido calculadas grá. fica y analíticamente; en particular, las líneas paralelas de tensión nula y varia. bles según las condiciones de carga y de la inclinación de la estructura.

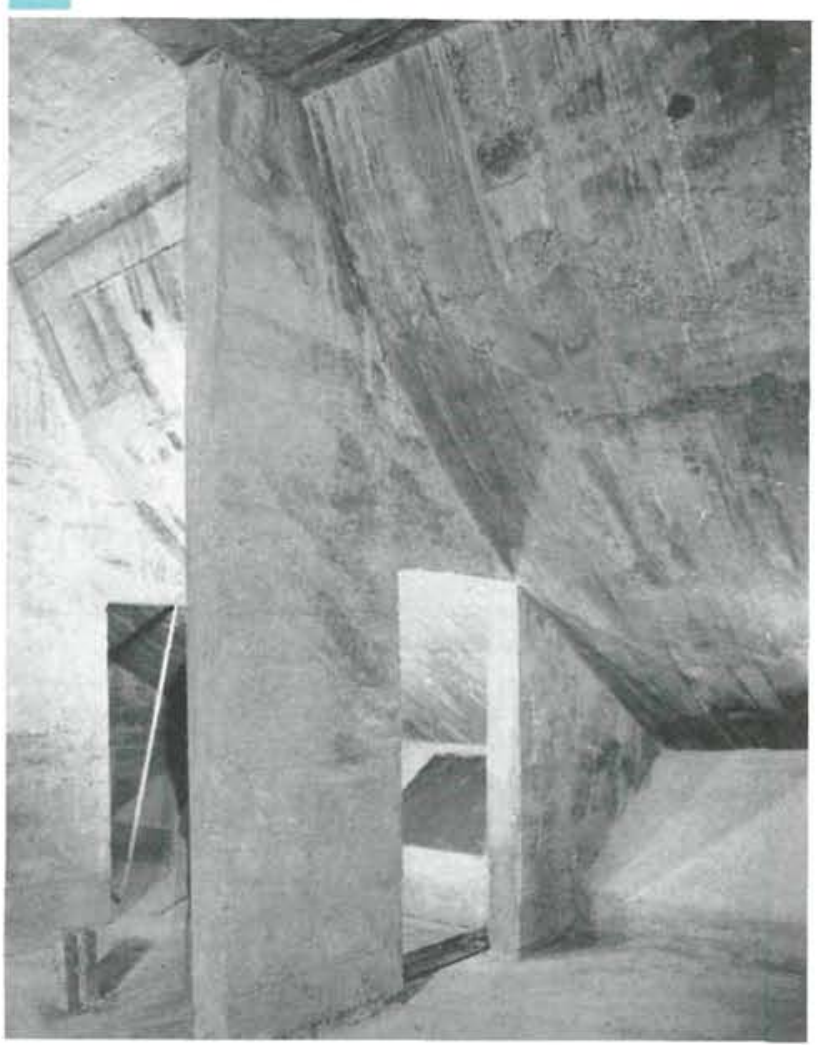




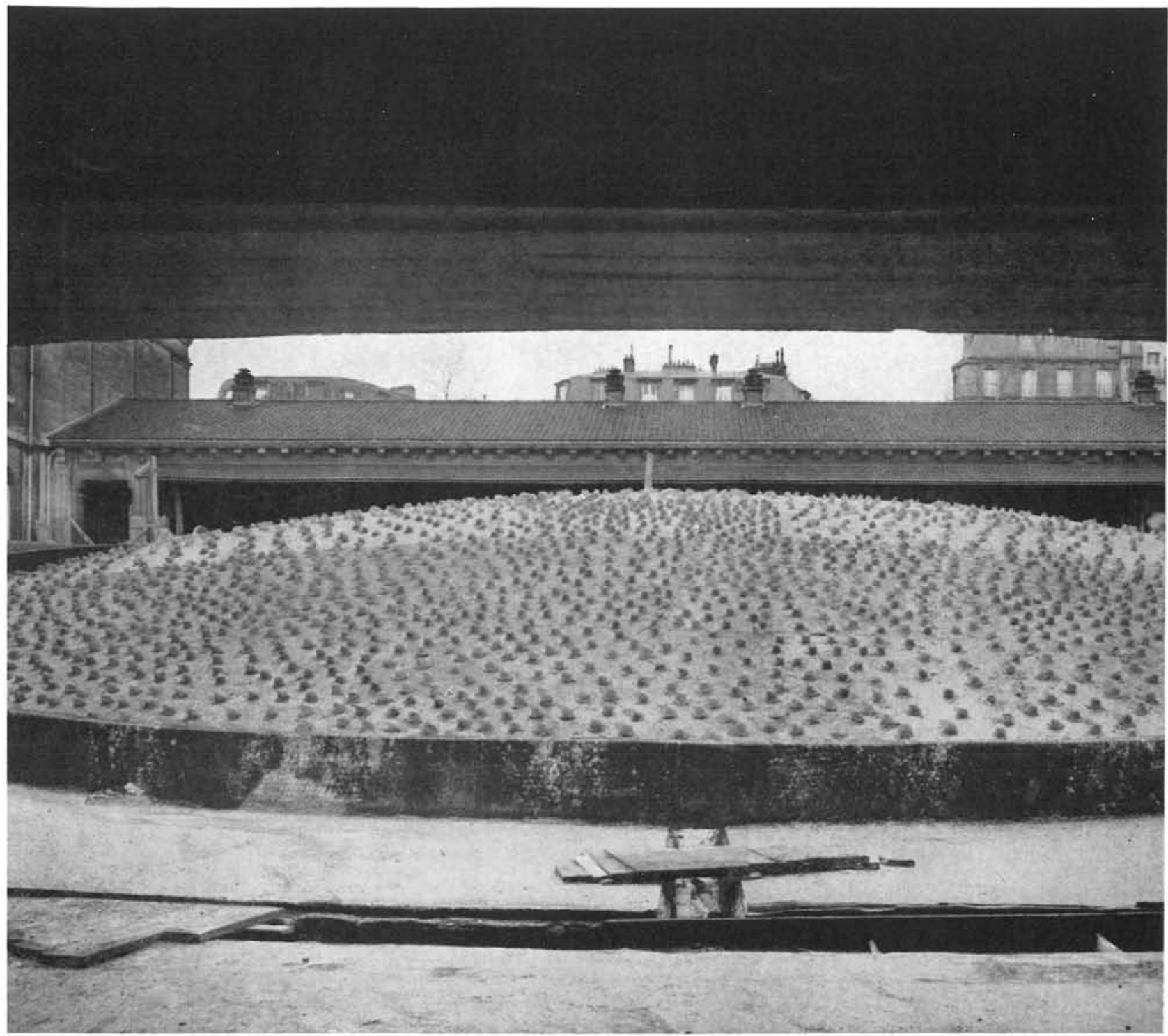

El empuje total es del orden de $510 \mathrm{t}$. El cálculo de la repartición del empuje según los paralelos se ha efectuado cortando la obra en un cierto número de anillos elementales y estudiando la iteración ejercida entre ellos a uno y otro lado del paralelo considerado.

La repartición llega a su máximo en la zona inferior de la unión de la bóveda esférica superior con la zona tórica periférica. El gabinete de estudios "Veritas" se ha encargado del control de la estructura durante la ejecución.

Ejecución.-Como no se disponía de espacio para el libre movimiento e instalaciones auxiliares, el hor migón se transportó por medio de canalizaciones de aire comprimido, dotadas de gran movilidad y con capacidad suficiente para abastecer al ritmo que este tipo de obras exige.

Los encofrados para el hormigonado de la zona perimetral tórica han requerido un serio estudio, constituyendo, por sí misma, la parte más delicada del hormigonado. Estos encofrados son de madera, reforzados con angulares metálicos y terminados con placas de madera contrachapada que proporcionaron superficies lisas y de buen aspecto después de desencofrar.

Los encofrados de la zona tórica se colocaron con ayuda de un mástil provisto de vientos, móvil, y ayudándose de un torno. Este aparejo de elevación permitía el transporte, colocación y ajuste de los encofrados

El hormigonado de la parte superior o bóveda se realizó siguiendo los procedimientos convencionales; sin embargo, se intentó adoptar sistemas nuevos y hormigonar por sectores, pero esta solución se complicaba tanto que fue preciso abandonarla.

Es de notar el efecto que producía al personal los entramados tubulares, ya que éstos tenian sus montantes según la normal al plano diametral inclinado, lo que daba lugar a perder el sentido de la vertical para recobrar el equilibrio. 


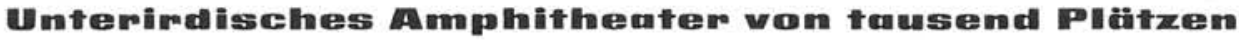

J. M. Hereng, Ing. Conseil.

Louis, Luc und Thierru Sainsaulieu, D. P. L. G., Architekten.

Das Konservatorium der Künste und Handwerke von Paris brauchte ein Amphitheater mit tausend Plätzen, das Stunden und Konferenzen dienen sollte. Aus Platzmangel entschloss man sich, es unterirdisch unter einem der Höfe zu bauen.

Die angenommene Lösung war die eines Elipsoiden mit Umdrehung der grösseren geneigten Achse bezüglich der wagerechten mit $32 \mathrm{~m}$ Durchmesser und $10 \mathrm{~m}$ max. Höhe; die Dicke in dem unteren Teile beträgt $50 \mathrm{~cm}$ und in dem oberen $20 \mathrm{~cm}$.

Diese Lösung ist von besonderem Interesse, da sie zwei Probleme auf einmal erledigte: das der Fundierung, das allgemein alle Gelände der Aufschüttung aufwerfen, und als weiteres, dass es den umstehenden Gehäusen und Gebäuden keine Belichtung wegnimmt. Die Last auf dem Boden ist stets geringer als $380 \mathrm{~g} / \mathrm{cm}^{2}$, selbst bei der zufälligen Annahme einer örtlichen Setzung in irgendeinem Teile der Stützung von $20 \mathrm{~m}$ Durchmesser. Der Transport des Betons erfolgte durch Pressluft - Kanal - Anlagen, um den Rhythmus des Betonierens nicht zu hemmen. Die Schalungen wurden sorgfältig studiert, besonders die der Uebergangszone zwischen dem Boden und dem Gewölbe, das den Hörsaal bedeckt.

Die Rohrgefüge, die die Schalungen des genannten Gewölbes stützen, wurden auf Grund von normalen Pfosten in der geneigten Durchmesserfläche montiert. Diese eigenartige Anordnung verursachte, dass das Personal, das sich auf ihnen bewegte, oft das Gefühl der Senkrechten verlor und damit das des Gleichgewichtes ihrer eigenen Person.

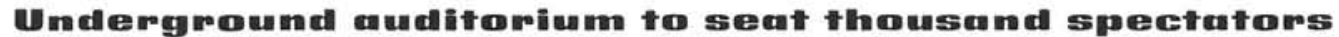

J. M. Hereng, civil engineer.

Louis, Luc and Thierru Sainsaulieu, D. P. G. L., architects.

The Arts and Crafts School of Paris was in need of an auditorium, with accommodation for one thousand persons, suitable for lessons and lectures. Owing to lack of space, it was decided to build it underground, below one of the courtyards.

The choice of roof shape was an ellipsoid of revolution, whose major axis is inclined to the horizontal. The maximum diameter is $32 \mathrm{~ms}$ and maximum height is $10 \mathrm{~ms}$. The thickness of the roof structure is $50 \mathrm{cms}$ along the lower part, and $20 \mathrm{cms}$ near the crown.

This solution was of special interest as it overcame simultaneously two difficulties; namely, the foundation, when this has to be based on fill soil, and the non-interference with the illumination of nearby buildings. In this case the loading on the soil is always less than 380 grams per $\mathrm{cm}^{2}$, even assuming a local settlement of the soil over any area of $20 \mathrm{~ms}$ in diameter.

Concrete has been transported to the required sites by means of compressed air chutes, with the result that the rate of concreting has not been interfered with. Formwork has been carefully thought out, especially over the zones where the floor surface changes into the auditorium vault.

The tubular structure supporting the formwork of the vault has been erected with columns which are normal to the inclined diametral plane of the surface of revolution. Owing to this unusual arrangement, the staff working at the site have often lost their sense of balance.

\section{Amploithécitrme soutremrouin de mmille places}

J. M. Hereng, Ingénieur-Conseil.

Louis, Luc et Thierru Sainsaulieu, D. P. L. G., architectes.

Le Conservatoire des Arts et Métiers de Paris avait besoin d'un amphithéâtre de mille places pour classes et conférences. Pour manque d'espace on décida de le construire sous l'une des cours.

La solution adoptée a été celle d'un ellipsoïde de révolution d'axe majeur incliné par rapport à l'horizontale, de $32 \mathrm{~m}$ de diamètre et $10 \mathrm{~m}$ de hauteur maximum; lépaisseur de la partie inférieure est de $50 \mathrm{~cm}$ et de la supérieure de $20 \mathrm{~cm}$.

Cette solution est d'un intérêt tout spécial, parce qu'elle a résolu deux problèmes à la fois: celui des fonda. tions, posé en général par tous les terrains de remblai et celui de respecter l'éclairage des édifices environ. nants. La charge au sol est toujours inférieure à 380 grammes par centimètre carré, même dans le cas supposé d'un affaissement local sur quelque zone d'appui de $20 \mathrm{~m}$ de diamètre. Le transport du béton a été réalisé à l'aide de canalisations d'air comprimé afin de ne pas interrompre le cours du bétonnage. Les coffrages ont été soigneusement étudiés, notamment ceux de la zone de transition entre le sol et la voûte qui couvre l'auditorium.

Les charpentes tubulaires qui soutiennent les coffrages de cette voûte ont été montées à l'aide de montants normaux au plan diamétral incliné. Cette disposition curieuse a fait que le personnel qui y travaillait perdait souvent le sens de la verticalité et celui de leur propre équilibre. 